\title{
Identification of Agency through Virtual Embodied Interaction
}

\author{
Takafumi Sakamoto and Yugo Takeuchi \\ Graduate School of Informatics, Shizuoka University, \\ 3-5-1 Johoku, Naka-ku, Hamamatsu, Shizuoka 4328011 Japan \\ gs12018@s.inf.shizuoka.ac.jp, takeuchi@inf.shizuoka.ac.jp
}

\begin{abstract}
To examine the identification of "social actors," we created an experimental environment to observe how people interpret the behavior of others. Our experimental environment, which physically provided interaction between a human and a computer, was a media system that connected two sides of the experimental environment to a computer network. In our experiment task, participants used our system to determine whether the other party was a human or a computer. In this study, we regard the attribution of agency toward the behaviors of others as a sign of agency identification. Our experiment results suggest that the human identification of "social actors" is induced by the interaction between the target entity and the subjects.
\end{abstract}

\section{Introduction}

People have the ability to regard anything around them as "social actors." In this paper, we define "social actor" as an entity that possesses the intellectual ability to form a relationship with humans [1]. Of course, humans are the most common social entity that possesses intellectual ability and can form relationships with others. However, this idea is not always applied even though the "social actor" is a human. The identification of a "social actor" to a human depends on the individual's psychological attribution by which one identifies others as "social actors." This mental activity can be shown in urban situations where people ignore others and treat them as walking obstructions. The identification and attribution of "social actors" are determined, not on their properties but on their actual behaviors during interaction.

The above analysis suggests the following question: How does interaction with others affect their identification when they bestow the label "social actor" on others? We carried out a simple psychological experiment to explore this question.

\section{Social Actor}

\subsection{Communicable Relationships}

We define a "social actor" as an entity with agency that can form a relationship with humans to exchange significant information. 
Agency is the property of an agent and contains the following features [2]:

\section{Intentionality}

The agent behaviors are purposive. The agent aims to achieve its purpose.

\section{Autonomy}

The agent behaves autonomously to achieve its purpose.

\section{Rationality}

The agent rationally and suitably behaves to achieve its purpose in any situations.

\section{Individuality}

The agent exists individually and acts respectively.

\section{Interactivity/Sociality}

The agent interacts with others to effectively achieve its purpose.

To form a relationship to communicate between humans and agents with such features, humans must understand the purpose behind an agent's behavior. However, such human understanding does not need to be consistent with the agent's actual inner status. The purpose of the agent's behavior, which the human supposes under this impression, does not necessarily agree with the agent's actual mind when the human is directly interacting with the agent.

In this paper, the concept of communication is different from the concept of interaction. Interaction means a state where mutual action is organized with or without understanding the purpose or the intention of an agent's behaviors. Communication denotes a state that can interpret the agent's behavior based on the understanding of an action's purpose and intention after assuming the establishment of interaction with it.

\subsection{Communication with Social Actor}

When an interaction partner's identity is unknown and provides no visual or auditory clues, such as appearance, do people identify the partner as a "social actor"? Suppose they identify such an unknown entity as a "social actor." What kind of interaction will be carried out between the two participants? In other words, when people identify the unknown entity as a "social actor" and form a relationship to communicate with it, what kind of interaction will be carried out? To explore this research issue, people interacted with unknown entities to solve the following problems:

1. Does the interaction partner possess agency?

2. When the interaction partner is an agent that possesses agency, what is the purpose of the agent's behaviors?

3. How would the agent's behavior be interpreted to achieve its purpose? 
Communication with the agent is established in phase (3), indicating that people can interpret agent behaviors based on an understanding of their purpose and intention.

\section{Experiment}

\subsection{Purpose}

In this section, we explain our experiment that examined whether people identified the agency through a simple interaction environment, which was described in Section 2.2, as the first phase of agency observation. We expressed a human whose identity and agency were unknown as a shadow that virtually reflected its physical position and movement to the participant. Then we observed the interaction between the participants and the shadow to explore whether agency identification occurred when the shadow's movements, which symbolized the movement of the unknown entity, interacted with the participant.

\subsection{Method}

We made two experimental conditions and equally assigned 30 university students to each condition. The only instruction given to them was that they were allowed to move freely within the $1.2 \times 0.9$ m floor.

In the human condition, the human acts as an unknown entity and is imaged as a 20-cm diameter, circle-shaped shadow. The shadow's position corresponds to the position of the performer's waist, which was measured by Kinect sensors. As shown in Fig. 1, the performer's movements are reflected in a shadow that appears in the participant's room. The performer looks at the display that shows the participant's position and her own relative position.

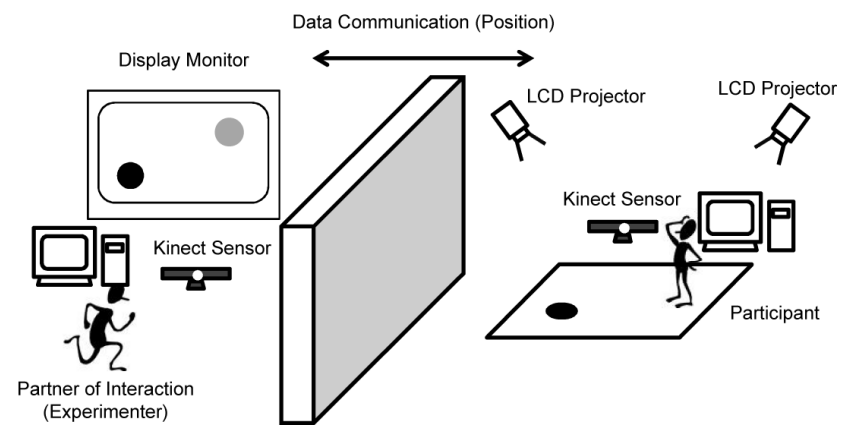

Fig. 1. Settings of the experiment 


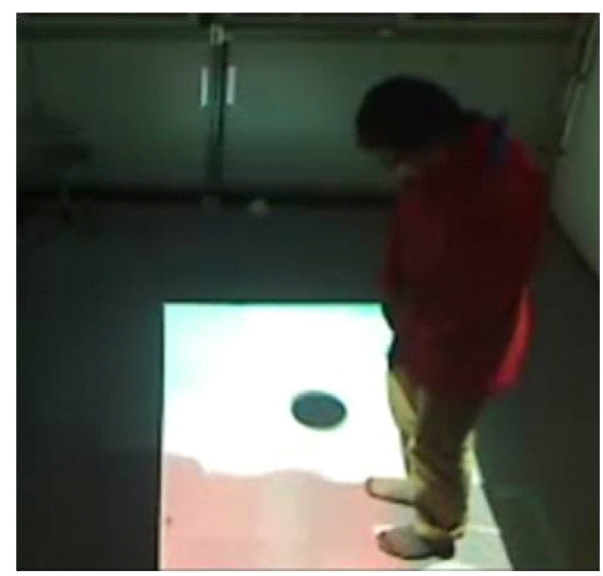

Fig. 2. Scene of interacting with the shadow

In the program condition, the human who is acting as an unknown entity in the human condition is supplanted by a computer program that determines whether the shadow is an unknown entity based on the current participant's position and decides its position with an algorithm and moves randomly between $0-800 \mathrm{~mm} / \mathrm{s}$.

The participants of each experimental condition freely interacted with the moving shadow for three minutes (Fig. 2) and then answered questionnaires.

\subsection{Observed Data}

We observed and analyzed the following data:

\section{A) Behavioral data}

- $\quad$ Log data of participant position (every 100ms)

- $\quad$ Log data of shadow position (every $100 \mathrm{~ms}$ )

- Interaction video

B) Questionnaires

- $\quad$ Participant impressions about the agency (Human / Computer / Undistinguished)

- $\quad$ Participant impressions about the shadow (animacy / intentionality)

\subsection{Results}

Fig. 3 shows the results of the participant impressions about the agency through questionnaires after the interaction. Nine of the fifteen participants in the human condition could not identify the agency by observing the shadow behaviors. Four of the fifteen participants thought that the shadow behaviors were controlled by a computer program. Only two participants identified the agency from the shadow behaviors. 


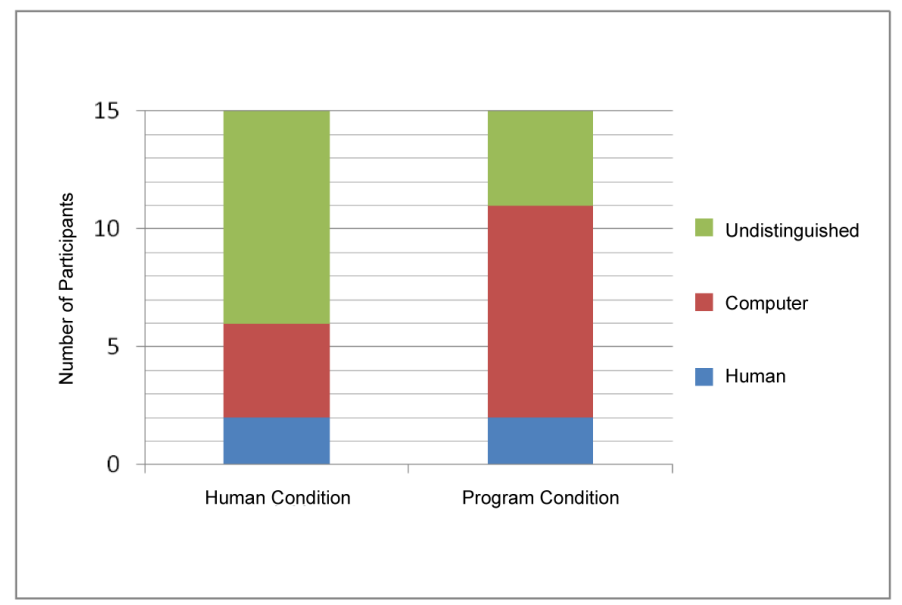

Fig. 3. Result of identification

The results of the program condition showed that nine of the fifteen participants correctly realized that the shadow behaviors were controlled by a computer program. Two participants identified the agency, and four participants could not identify it by observing the shadow behaviors.

Fig. 4 shows the number of movements in each case of the participant impressions concerning the agency. We found significant results when the participants identified the agency (human condition). They actively moved and interacted with the shadow.

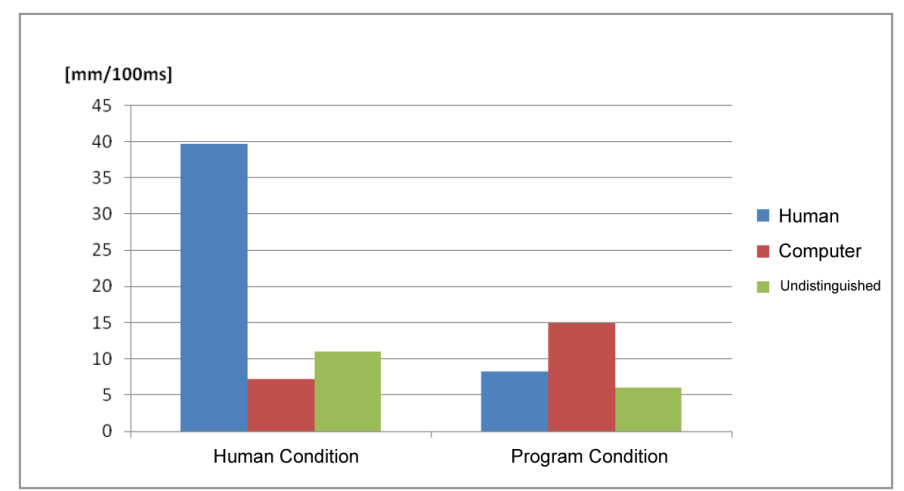

Fig. 4. Result of behavioural data 


\subsection{Considerations}

As shown in the human condition results, the participants who identified the agency of the shadow significantly recognized the shadow behaviors and actively interacted with it, although their number was few. They correctly identified that their interaction partner possessed agency, indicating that they not only visually observed the shadow behavior but also physically and actively interacted with the shadow to verify the agency (Section 2.1).

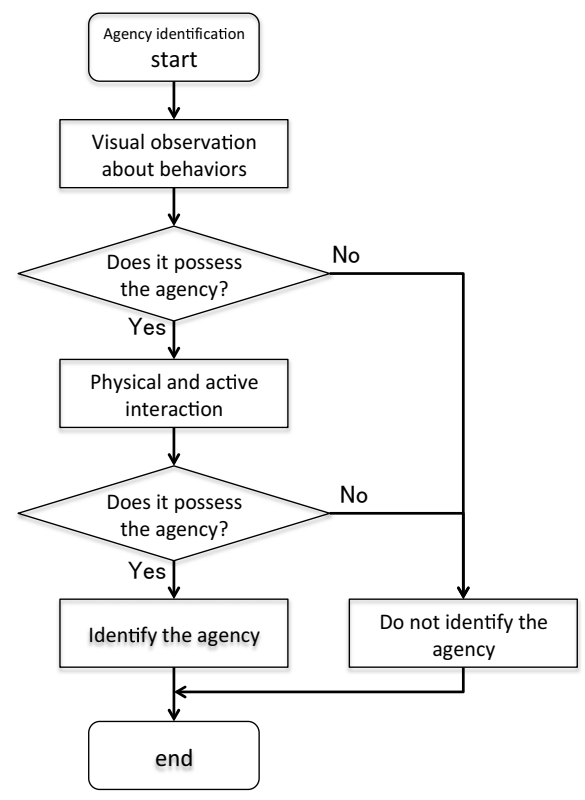

Fig. 5. Flow of agency identification

On the other hand, as shown in the results of the program condition, most participants did not identify the agency through the shadow behaviors. They assumed that the shadow movement was controlled by a computer program. This supposition was carried out by limited interaction and visual observation, not by physical and active interaction.

Agency might be identified as the following process (Fig. 5).

1. The participants carried out agency identification by visual observation.

2. If they intuitively identified the agency, they interacted more actively to verify their supposition that their interaction partner possesses agency.

3. If they did not identify agency, they believed that their interaction partner was a machine. 


\section{Conclusion}

In this study, we examined whether people can identify agency through a simple interaction environment. Our result indicates that agency identification is actively achieved by observation and interaction steps.

\section{References}

[1] Fogg, B.J.: Persuative Technology: Using Computers to Change What We Think and Do. Morgan Kaufmann Publishers (2003)

[2] Dennett, D.C.: The Intentional Stance. MIT Press (1987) 